\title{
BMJ Open Global research trends in spinal ultrasound: a systematic bibliometric analysis
}

\author{
Xiao Zhai, ${ }^{1}$ Jin Cui, ${ }^{1,2}$ Jie Shao, ${ }^{1}$ Qijin Wang, ${ }^{3}$ Xiao Chen, ${ }^{1}$ Xianzhao Wei, ${ }^{1}$ \\ Xiaoyi Zhou, ${ }^{1}$ Ziqiang Chen, ${ }^{1}$ Yushu Bai, ${ }^{1}$ Ming Li ${ }^{1}$
}

To cite: Zhai X, Cui J, Shao J, et al. Global research trends in spinal ultrasound: a systematic bibliometric analysis. BMJ Open 2017;7:e015317. doi:10.1136/ bmjopen-2016-015317

- Prepublication history and additional material for this paper are available online. To view these files, please visit the journal online (http://dx.doi org/10.1136/bmjopen-2016015317).

$\mathrm{XZ}, \mathrm{JC}$ and JS contributed equally.

Received 29 November 2016 Revised 3 August 2017 Accepted 4 August 2017

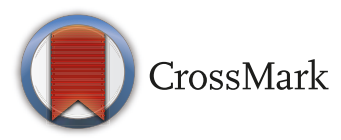

${ }^{1}$ Department of Orthopedics, Changhai Hospital, Second Military Medical University, Shanghai, China

${ }^{2}$ Graduate Management Unit, Changhai Hospital, Second Military Medical University, Shanghai, China

${ }^{3}$ Department of Endocrinology, Changhai Hospital, Second Military Medical University, Shanghai, China

Correspondence to

Dr Ziqiang Chen;

ziqiang_chen81@126.com

\section{ABSTRACT}

Background In recent years, there has been increased interest in the use of ultrasound technology in the evaluation of spinal and paraspinal regions.

Objective This study aimed to investigate trends in spinal ultrasound research from 1994 to 2015 and compare the contributions of such research from different countries and authors.

Study design Bibliometric analysis.

Setting Publications related to spinal ultrasound from 1994 to 2015 were retrieved from the Web of Science database.

Methods Excel 2013, GraphPad Prism 5, and V0Sviewer were used to summarise bibliometric features, including the number of publications, citation frequency, $\mathrm{H}$-index, and country contributions and hotspots (keywords of popular scientific fields).

Results A total of 3859 papers were included. The global inflection point (the point in time when the publication growth rate moved from positive to negative) came in 2010. The United States contributed the largest percentage of articles $(1041 ; 26.9 \%)$, with the most citations (19 848) and the highest H-index (61). The journals Osteoporosis International and Spine had the highest publication number. The University of Toronto and the University of California, San Francisco were the most contributive institutions. Studies could be divided into three clusters: surgery, osteoporosis, and others. The keywords 'adolescent idiopathic scoliosis' and 'anaesthesia' were the latest hotspots, appearing around 2012.

Conclusion Spinal ultrasound literature has grown continuously over the last 22 years, with the rate slowing down after 2010. The United States was the largest contributor in this field. Recent studies on topics related to 'adolescent idiopathic scoliosis' and 'anaesthesia' were relatively new and should be closely followed in spinal ultrasound research.

\section{INTRODUCTION}

Spinal disease is one of the most common causes of pain. It is estimated that about $25 \%$ of postmenopausal women suffer from osteoporotic compression fractures of the spine, ${ }^{1}$ and about 10000 new cases of spinal cord injury are reported every year. ${ }^{2}$ Ultrasound (US) technology has emerged as a medical

\section{Strengths and limitations of this study}

This bibliometric study included data on publication number, citation frequency, relative research interest, mean number of citations per paper, and $\mathrm{H}$-index.

- The fitting mathematical model was used to analyse the time trend of the publications by using GraphPad Prism 5 .

- VOSviewer, a Java programme, was used to visualise and analyse hotspots, masterpiece clusters, and cocitation networks.

- The fitting mathematical model might be improved in the future, since the increasing trend in the number of publications might extend beyond what is expected according to the proposed model.

device for evaluating spinal and paraspinal regions. ${ }^{3}$ Quantitative US has been widely used for osteoporosis assessment and fracture risk estimation in peripheral anatomical sites (eg, the heel) ${ }^{4}$ because of its ability to visualise muscles, tendons and internal organs, capturing their size, structure and pathological lesions. ${ }^{5}$ The method recently reported by Conversano et al in 2015 indicated that the osteoporosis score obtained via echographic approaches could be an accurate diagnostic parameter for spinal osteoporosis diagnosis. ${ }^{6}$ Since the 1980s, US has also been applied as an intraoperative navigation tool. ${ }^{7}$ In recent years, there has been an increase in the use of US for spinal anaesthesia. ${ }^{8}$ However, this rapid progress in spinal US is underestimated; therefore, it is necessary to summarise the current status of spinal US research and explore promising keywords.

Bibliometrics is a viable means to quantitatively and qualitatively evaluate trends in research activity over time using literature databases and literature metrology characteristics. It provides a way to grasp development in a certain field and evaluate academic groups and individual researchers. ${ }^{9}$ Bibliometric studies can also provide supporting 
evidence for policy and decision making. ${ }^{10}$ Such bibliometric studies have progressed in many scientific fields, including diabetes, ${ }^{11}$ cardiovascular disease,${ }^{12}$ respiratory medicine ${ }^{13}$ gastrointestinal diseases ${ }^{14}$ and exosome studies. ${ }^{15}$

This study investigated trends in spinal US research in recent years. Since the Web of Science began collecting related papers in 1994, we analysed publication outcomes from the last 22 years (1994-2015). This first attempt to use bibliometrics to analyse spinal US related publications aims to provide a better understanding of global trends in the application of spinal US.

\section{METHODS}

\section{Data source and search strategy}

Bibliometric analysis was performed using the Web of Science (WoS) and Essential Science Indicators (ESI) databases, including the Science Citation Index Expanded (SCIE) database.

Search terms were based on MESH terms on PubMed. In this study, the search terms were as follows: theme $=$ ((ultrasound* OR ultrasonography OR ultrasonic) AND (spine OR spinal OR vertebra OR vertebrae)) AND publishing year $=(1994-2015)$.

We refined the search for certain countries or regions by selecting country/region in the databases. The top 20 productive countries/regions are described in this research.

\section{Information extraction}

The data were downloaded from the WoS database and imported into Microsoft Excel 2013. Two researchers (XZ and JC) independently verified the data entry and collection. The entered data were listed as follows: publication number, citation frequency, relative research interest, mean number of citations per paper, H-index, ${ }^{16} 17$ impact factors of journals, and funding sources. Differences between the two researchers' verifications were discussed to reach consensus. Finally, the two researchers manually cleaned and analysed the data in Excel. The most recent Central Intelligence Agency (CIA) statistics on population sizes were used in this study. ${ }^{18}$

Relative research interest was designed as the quantity of weighted publications per year divided by the number of publications across all disciplines per year. ${ }^{19}$ The H-index is an author-level metric that attempts to measure both the productivity and citation impact of publications by a scientist or scholar. ${ }^{19}$ Specifically, H-index means he or she has $\mathrm{H}$ papers, each of which has been cited more than $\mathrm{H}$ times. For example, if a researcher has five publications, $\mathrm{A}, \mathrm{B}, \mathrm{C}, \mathrm{D}$ and $\mathrm{E}$, with $10,8,5,4$ and 3 citations, respectively, the H-index is equal to 4 since the fourth paper has 4 citations and the fifth has only 3 . In contrast, if the same studies have $25,8,5,3$, and 3 citations, then the index is 3 since the fourth paper has only 3 citations. ${ }^{20}$

\section{Statistical analysis}

Microsoft Office Excel 2013 (Microsoft Corporation, Santa Rosa, California, USA) was used to analyse the characteristics of the publications, including publication number, relative ratio, country, institution, funding institution, citation frequency, and $\mathrm{H}$-index.

The time trend of the publications was analysed by fitting mathematical models using GraphPad Prism 5 (GraphPad Software Inc., California, USA). Given its good fitness and ability to predict future trends in the literature,,$^{21} 22$ the logistic growth model $f(x)=c /\left[1+a \times e^{-b \times(x-1994)}\right]$ was used to model the cumulative volume of documentation. The symbol $x$ represented the year, and $f(x)$ was the cumulative volume of papers by year. The point in time when the publication growth rate moved from positive to negative is referred to as the inflection point of the logistic growth curve, which was generated using the formula $T=\operatorname{In} a / b{ }^{22}$

The Java programme VOSviewer (Leiden University, Leiden, The Netherlands) is used for the bibliometric visualisation and analysis of the literature. ${ }^{23}$ In this research, it was used for co-citation network analysis and visualisation. Hotspots, defined as the keywords of popular scientific fields, were calculated and visualised using VOSviewer according to keyword frequency in titles and abstracts. Clusters of masterpieces in research fields were also visualised based on citation frequency in the WoS data.

\section{RESULTS}

\section{Evaluation of global publications}

A total of 3859 papers were identified. In general, the number of publications per year indicated a steadily increasing trend over the past 22 years (figure 1A). Figure $1 \mathrm{~B}$ shows the model fitting curves of the growth trend. The global inflection point (the point in time when the publication growth rate moved from positive to negative) occurred in 2010.

The top 20 countries with the most publications are shown in table 1. The United States published the most papers $(1041 ; 26.98 \%)$, followed by Germany (368 papers; $9.53 \%)$. After adjusting for population, Switzerland had the most publications per one million people (60.63), followed by Austria (11.36).

\section{Citation frequency and $\mathrm{H}$-index analysis}

The citation frequencies and H-indexes of the top 20 countries are shown in table 1 . Papers from the United States had the highest citation frequencies $(19,848)$ and H-indexes (61). The UK ranked second in citation frequency.

Highly contributive institutions, funding bodies and journals The top 10 contributive institutions are listed in figure 2A. The University of Toronto published the most papers (89 papers, 1482 citations, 21 H-index), and the University of California, San Francisco (UCSF), ranked second (54 
A

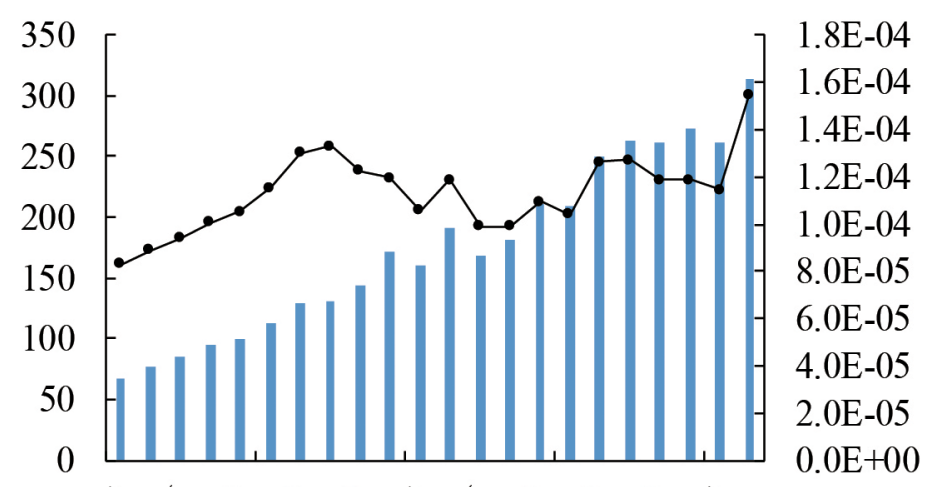

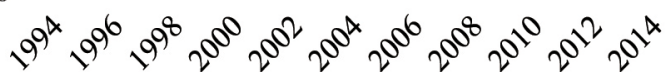

Publications $\rightarrow$-Relative Research Interest
$\mathrm{B}$

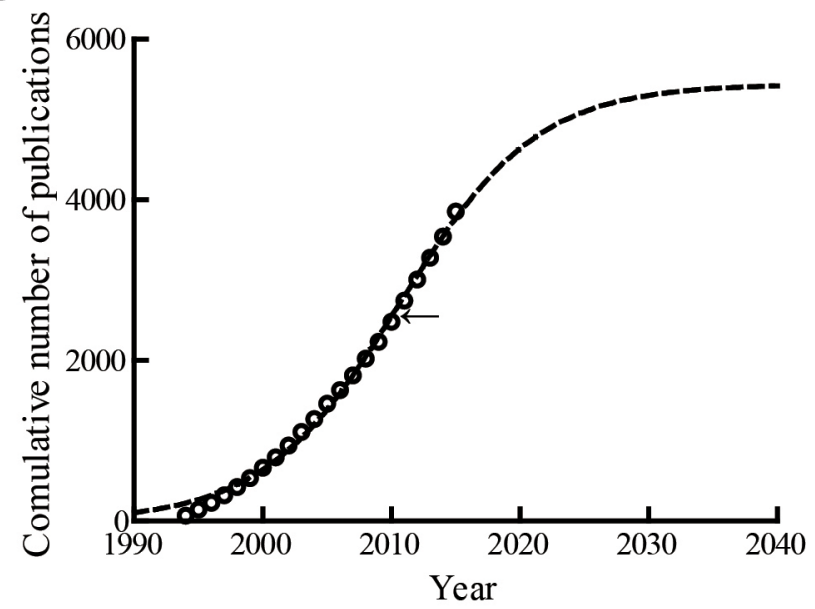

Figure 1 Global trends in research on spinal ultrasound (US) applications. (A) The number of worldwide publications on spinal US applications and the line of the sum of research-related article fractions (percentage of research from all regions). (B) Model fitting curves of growth trends in worldwide publications on spinal US applications.

papers, 2047 citations, $24 \mathrm{H}$-index). In contrast, papers from UCSF had the highest citations and H-indexes (54 papers, 2047 citations, 24 H-index).

The top 10 funding bodies are shown in figure 2B. Altogether, 31 studies were funded by the National Institutes of Health (NIH) (ranked first), and 16 were funded by the National Natural Science Foundation of China (NSFC) (ranked second). The Swiss firm Novartis was the top private funding institution, supporting 13 studies.
The top 10 journals for spinal US application are listed in figure 2C. Osteoporosis International published the most (165 papers), and Spine ranked second (79 papers).

\section{Hotspot analysis}

Figure 2D shows the research field distribution of publications related to spinal US application. The most popular research fields were neuroscience and neurology,

Table 1 Publications in the most productive countries

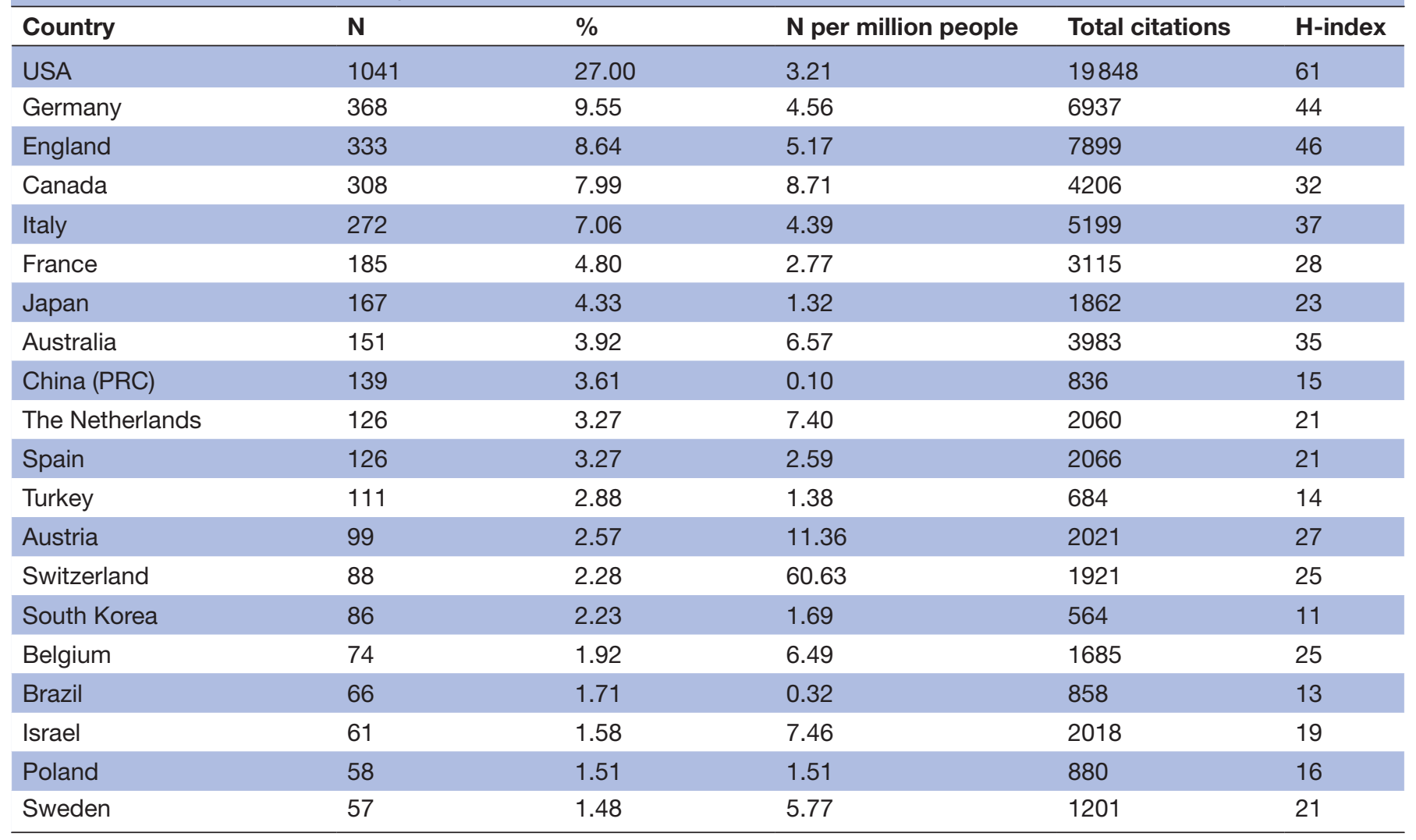


A

$\begin{array}{llllll}0 & 20 & 40 & 60 & 80 & 100\end{array}$

\begin{tabular}{|c|c|}
\hline UNIV TORONTO & $-21^{30}$ \\
\hline UNIV CALIF SAN FRANCISCO & $\begin{array}{ll} & 44 \\
\end{array}$ \\
\hline HARVARD UNIV & $21^{27}$ \\
\hline UNIV BRITISH COLUMBIA & -711 \\
\hline UNIV QUEENSLAND & $2132^{37}$ \\
\hline UNIV SHEFFIELD & $13^{21} 35$ \\
\hline UNIV PITTSBURGH & $15^{16} 34$ \\
\hline UNIV WASHINGTON & $-13^{16} 33$ \\
\hline MAYO CLIN & -511 \\
\hline UNIV ALBERTA & 6 \\
\hline
\end{tabular}

B

0

50

$\square$ Sum of publication $\quad$ Sum of citation $(\times 100) \quad$ h-index
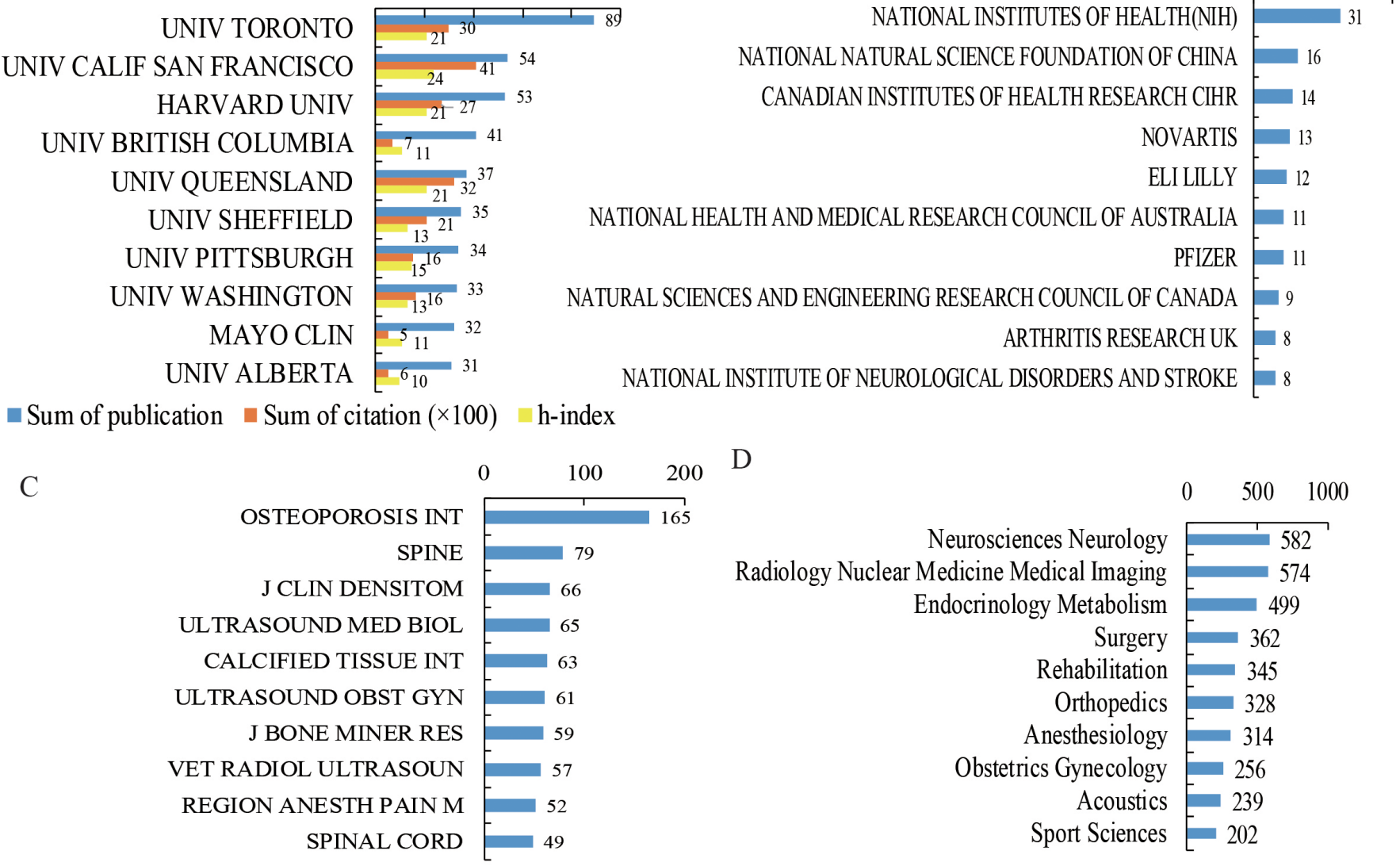

D

\begin{tabular}{rlr} 
& 500 & 1000 \\
Neurosciences Neurology & 582 \\
\cline { 2 - 2 } Radiology Nuclear Medicine Medical Imaging & 574 \\
Endocrinology Metabolism & 499 \\
Surgery & 362 \\
Rehabilitation & 345 \\
Orthopedics & 328 \\
Anesthesiology & 314 \\
Obstetrics Gynecology & 256 \\
Acoustics & 239 \\
Sport Sciences & 202
\end{tabular}

Figure 2 (A) The sum number, citation frequency and $\mathrm{H}$-index data of publications on spinal ultrasound (US) applications from the top 10 contributing institutions. (B) The number of studies funded by the top 10 funding institutions. (C) The number of publications in the top 10 journals on spinal US applications. (D) The number of publications in the top 10 popular fields of research on spinal US applications.

radiology, nuclear medicine and medical imaging, and endocrinology and metabolism.

Bibliometric maps generated by VOSviewer show the keyword analyses. According to the maps (figure 3A), studies were divided into three clusters: surgery (left corner in red), osteoporosis (right corner in green), and others (middle corner in blue).

Figure 3B shows the time distribution of keywords. 'Adolescent idiopathic scoliosis' (AIS) in the third cluster (others) appeared as a keyword 45 times in 2012, leading the latest research points. In addition, keywords related to 'osteoporosis' appeared earlier than 'surgery' and others, and keywords related to 'anaesthesia', such as 'spinal block anaesthesia', appeared in recent years. Supplementary table 1 lists the detailed data used to generate the bibliometric maps.

\section{Cluster analysis of masterpieces in each research field}

Figure 4 shows the bibliometric map of co-citations for papers on spinal US application. Papers cited more than 10 times were divided into four clusters. The article titled 'Statistical methods for assessing agreement between two methods of clinical measurement', published in Lancet in 1986, was the most cited (229 citations). Supplementary table 2 shows the detailed data used to generate the bibliometric map.

\section{DISCUSSION}

Global trends in research on spinal US application

As demonstrated in our previous study, ${ }^{15}{ }^{19}$ bibliometric and visualised analysis can show the current status and make future predictions. Therefore, the present study used the same method to evaluate spinal US applications with regard to contributing countries, institutions, funding institutions and research focuses. ${ }^{24}$

Given the situation of ageing populations in most developing countries, the number of patients with osteoporosis is expected to increase in the coming years. ${ }^{25}$ To reduce exposure to X-ray radiation during examinations, hospitals are increasingly using ultrasonic devices. ${ }^{22}$ As shown in figure 1A, relative research interest peaked around 20002002, which could be mostly related to studies of osteoporosis, as shown in figure 3. Furthermore, improvements have emerged in ultrasonic technologies, ${ }^{26}$ such as the use of small US probes, and more spinal surgeons have become interested in using US in surgery and anaesthesia. ${ }^{27}$ Finally, with improvements in quality of life, ordinary people's 


\section{A Cluster 3: Others (physical therapy et. al)}

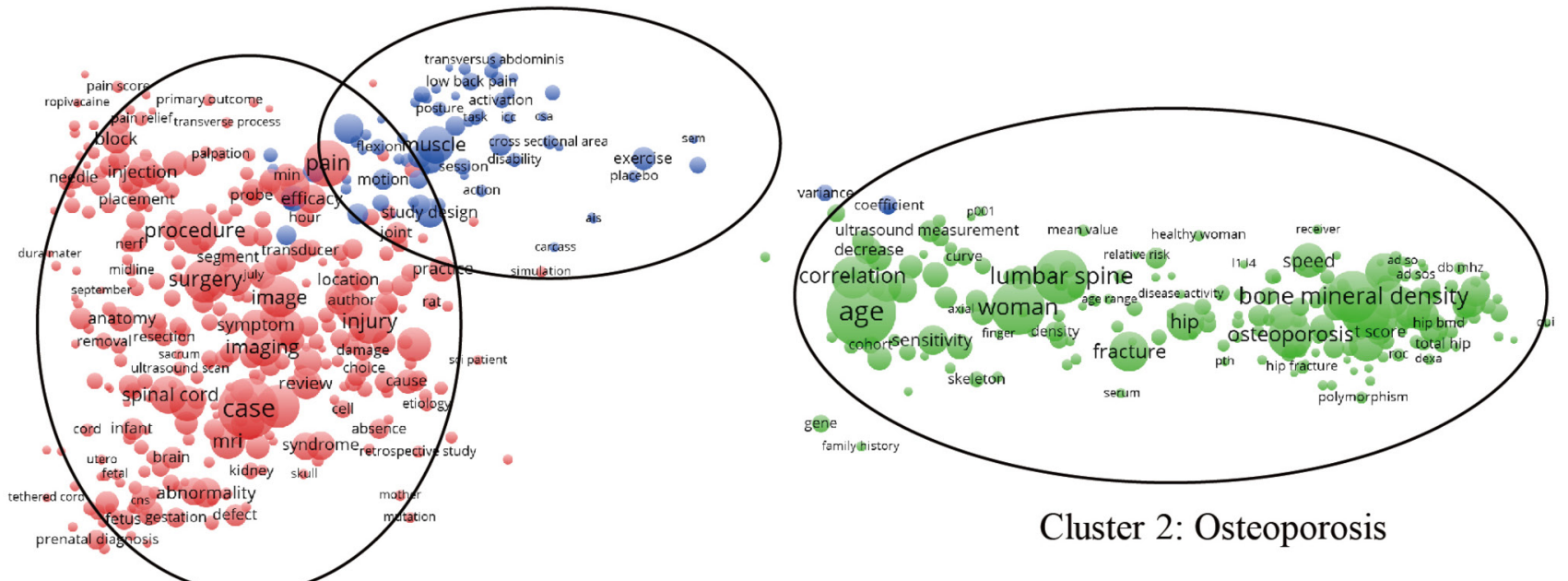

Cluster 1: Surgery

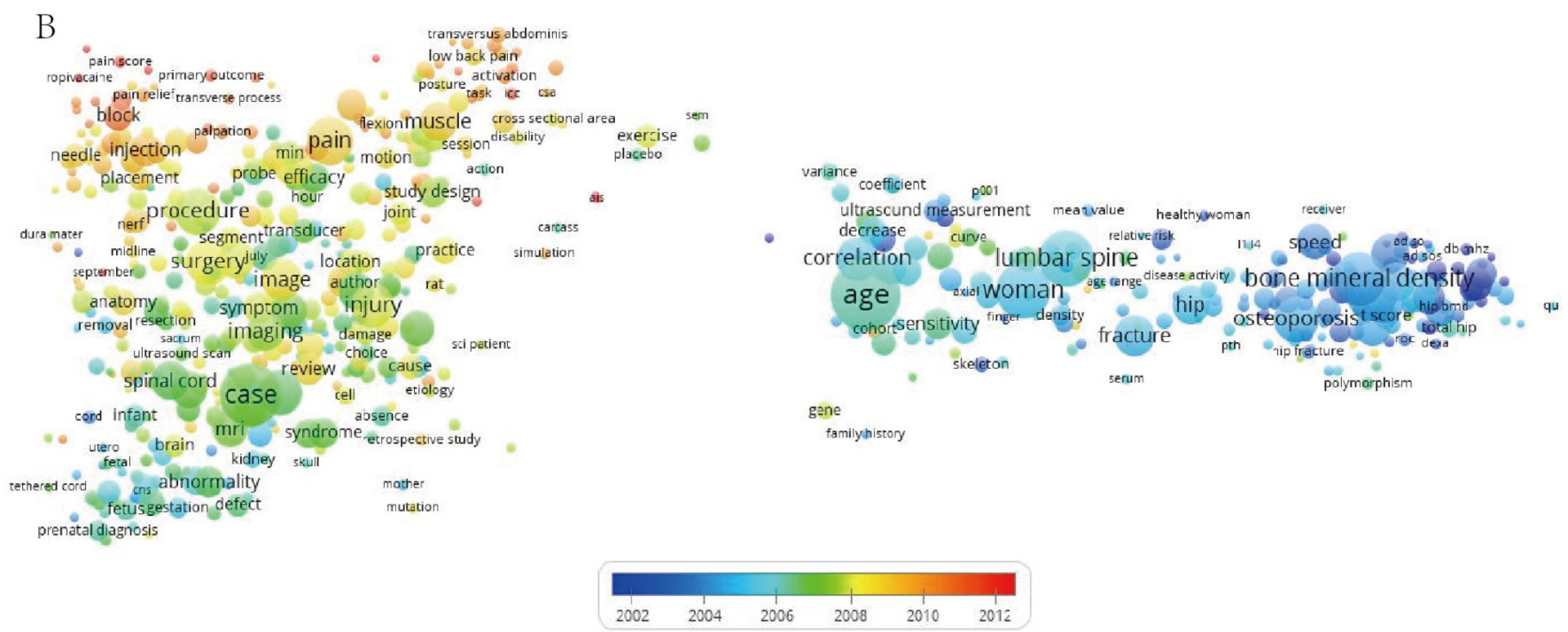

Figure 3 (A) Mapping of keywords in the research on spinal ultrasound (US) applications; the size of the points represents the frequency, and the keywords are divided into three clusters: surgery (left corner in red), osteoporosis (right corner in green), and others (middle corner in blue). (B) Distribution of keywords according to the mean frequency of appearance; keywords in blue appeared earlier than those in yellow.

awareness of the importance of health has gradually increased. More ultrasonic equipment for physiotherapy and rehabilitation has become commercially available. As a result, the number of research publications on spinal US has increased. Investigating bibliometric trends in spinal US helps us to better understand the history and future of spinal US application.

Although global research has shown an increasing trend, the regression growth model for the cumulative amount showed an inflection point in 2010, which means growth could become steady and slow. However, the possibility remains that the increasing trend will go on longer than anticipated by the proposed model because of breakthroughs in ultrasonic probe technologies and new imaging algorithms.
The United States was the leading country in both the quantity (total publication number) and quality (H-index) of research on spinal US application. In addition, most of the major contributing institutions were based in the United States and Canada. The listed institutions could potentially become partners for cooperation among those who are committed to this field. With the funding guarantees of the NSFC in China, top Chinese institutions are expected to emerge in the future. For journals, future development in this field may still be showcased in Osteoporosis International and Spine since they published the most articles in the past.

\section{Research focus on spinal US}

The network map indicated that osteoporosis was the dominant point in the early years of US research, during 


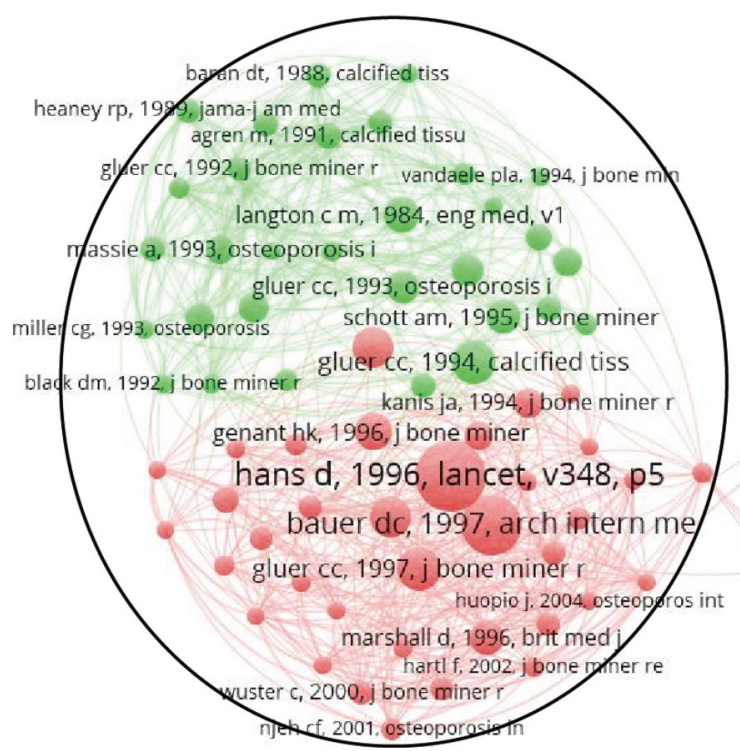

Cluster $1 \& 2$ : Osteoporosis

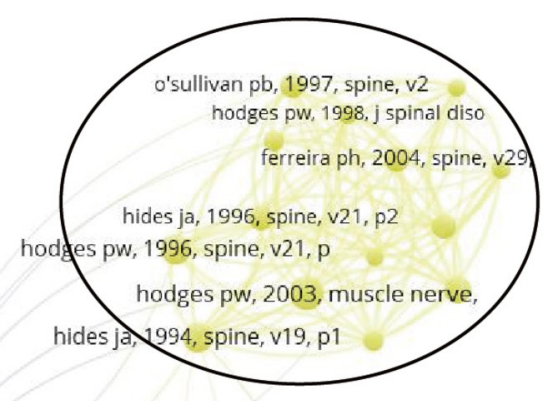

Cluster 3: Surgery

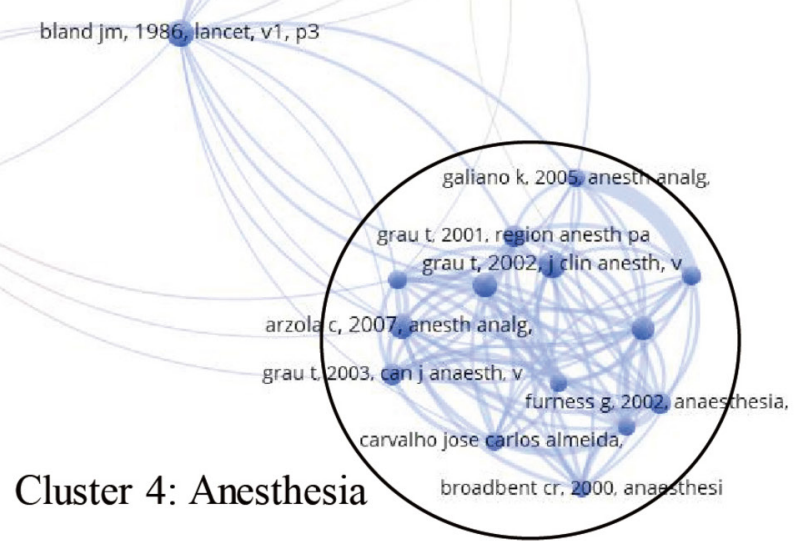

Figure 4 Mapping of co-cited references related to spinal ultrasound (US) application. (Note: given the large number of cited references, this study only selected papers cited more than 10 times for analysis. Ultimately, 680 papers were included in the analysis. The 680 points with different colours represent the 680 cited papers. A line between two points means that both were cited in one paper. A shorter line indicates a closer link between two papers. Points in the same colour belong to the same cluster among the four total clusters: clusters one and two (green and red) are papers related to osteoporosis; cluster three (yellow) contains papers related to surgery; and cluster four (blue) contains papers related to anaesthesia.)

which US was mainly used to determine bone density. Regarding the most cited papers, the study by Hans $e t a l^{28}$ published in Lancet, was first with 229 citations. That study showed that US could help predict the prevalence of hip fracture among older women by determining the bone mineral density (BMD) of calcaneus; moreover, it found no significant differences in the results between US and DPXA (dual energy X-ray absorptiometry). Meanwhile, a study by Bauer et al cited 179 times showed that broadband ultrasonic attenuation can help predict fractures in older women. ${ }^{29}$

Aside from osteoporosis, US application in spinal surgery has become a new research hotspot. A 2003 study by Hodges $e t a l^{30}$ showed that the isometric contraction of some muscles could be measured by ultrasonic imaging. Another study by Hodges et al in $1996^{31}$ showed that delayed transversus abdominis contraction indicates inefficient muscular stabilisation of the lumbar spine; the authors also predicted that US application to spinal block anaesthesia would become an important topic in the future, which proved to be true. A 2002 study by Grau et $a l^{32}$ showed that US application could significantly improve the quality of epidural anaesthesia. Finally, a 2007 study by Arzola $e t a l^{33}$ indicated that US application could improve the success rate of anaesthesia by helping find the appropriate entry point.
The word distribution in titles and abstracts indicated that AIS was the keyword with the highest frequency. Ungi et $a \vec{l}^{34}$ found that the kyphosis angle of patients with AIS could be measured effectively by ultrasonography, which was consistent with the results of Wang $e t a l^{55}$ and Young et $a l^{36}$ Finally, Manbachi et $a l^{37}$ reported that US could be used to guide pedicle screw implantation. ${ }^{22}$

\section{Strengths and limitations}

This bibliometric study provided information about research on US application to spinal regions. The reader can readily grasp the history of spinal US and find appropriate cooperative institutions or authors.

Although this study's data analysis was relatively comprehensive and objective, there are certain limitations. First, the SCIE database mainly includes literature in English and is lacking in non-English publications. Second, differences existed between the bibliometric analysis results and real research conditions. Some recently published high-quality papers might not be emphasised because of low citation frequency. Further, increasing publication trends might continue longer than suggested by the proposed model. Third, the data in this study are open, and new studies are published every day. 


\section{CONCLUSION}

The United States was the largest contributor to studies on the use of ultrasound in spinal applications. The journals Osteoporosis International and Spine had the most publications related to spinal US. The University of Toronto and UCSF are expected to be good candidates for collaborative research in this field. The application of US to AIS and anaesthesia could be the research hotspots to follow in the coming years.

Acknowledgements The authors would like to thank Professor Shunxing Zhang from the Department of Foreign Language Teaching at the Second Military Medical University, and professional editors at Editage, a division of Cactus Communications, for their help with the grammar and readability of this paper. This study was supported by the National Natural Science Foundation of China (81701199,81601953, 81670741), Science and Technology Commission of Shanghai Municipality $(16411971400,17441900500)$, Fund of the Second Military Medical University (2014QN15), and Fund of Changhai Hospital (CH201717).

Contributors XZ and JC researched the data and wrote the manuscript. ZC researched the data. $M L$ reviewed/edited the manuscript. JS and QW contributed to the discussion, $\mathrm{XC}$ and $\mathrm{YB}$ contributed to the discussion and reviewed/edited the manuscript. XW and $X Z$ contributed to the revision.

Competing interests None declared.

Provenance and peer review Not commissioned; externally peer reviewed.

Data sharing statement All additional data were attached in this system.

Open Access This is an Open Access article distributed in accordance with the Creative Commons Attribution Non Commercial (CC BY-NC 4.0) license, which permits others to distribute, remix, adapt, build upon this work non-commercially, and license their derivative works on different terms, provided the original work is properly cited and the use is non-commercial. See: http://creativecommons.org/ licenses/by-nc/4.0/

(C) Article author(s) (or their employer(s) unless otherwise stated in the text of the article) 2017. All rights reserved. No commercial use is permitted unless otherwise expressly granted.

\section{REFERENCES}

1. Kim DH, Vaccaro AR. Osteoporotic compression fractures of the spine; current options and considerations for treatment. Spine $J$ 2006;6:479-87.

2. McDonald JW, Sadowsky C. Spinal-cord injury. Lancet 2002;359:417-25.

3. American Academy of Neurology's TherapeuticsTechnology Assessment Subcommittee. Review of the literature on spinal ultrasound for the evaluation of back pain and radicular disorders: report of the Therapeutics and Technology Assessment Subcommittee of the American Academy of Neurology. Neurology 1998;51:343-4.

4. Roux C, Dougados M. Quantitative ultrasound in postmenopausal osteoporosis. Curr Opin Rheumatol 2000;12:336-45.

5. Moon SH, Park MS, Suk KS, et al. Feasibility of ultrasound examination in posterior ligament complex injury of thoracolumbar spine fracture. Spine 2002;27:2154-8.

6. Conversano F, Franchini R, Greco A, et al. A novel ultrasound methodology for estimating spine mineral density. Ultrasound Med Biol 2015;41:281-300.

7. Dohrmann GJ, Rubin JM. History of intraoperative ultrasound in neurosurgery. Neurosurg Clin N Am 2001;12:155.

8. Tsui B, Suresh S. Ultrasound imaging for regional anesthesia in infants, children, and adolescents: a review of current literature and its application in the practice of extremity and trunk blocks. Anesthesiology 2010;112:473-92.

9. Ekinci S, Agilli M, Ersen O, et al. Letter to the editor regarding analysis of changing paradigms of management in 179 patients with spinal tuberculosis during a 12-Year period and proposal of a new management algorithm. World Neurosurg 2015;84:2072-72.
10. Avcu G, Sahbudak Bal Z, Duyu M, et al. Thanks to trauma: a delayed diagnosis of Pott disease. Pediatr Emerg Care 2015;31:E17-E18.

11. Geaney F, Scutaru C, Kelly C, et al. Type 2 diabetes research yield, 1951-2012: bibliometrics analysis and density-equalizing mapping. PLoS One 2015;10:e0133009.

12. Shuaib W, Khan MS, Shahid $\mathrm{H}$, et al. Bibliometric analysis of the top 100 cited cardiovascular articles. Am J Cardiol 2015;115:972-81.

13. Seriwala HM, Khan MS, Shuaib W, et al. Bibliometric analysis of the top 50 cited respiratory articles. Expert Rev Respir Med 2015;9:817-24.

14. Narotsky D, Green PH, Lebwohl B. Temporal and geographic trends in celiac disease publications: a bibliometric analysis. Eur $\mathrm{J}$ Gastroenterol Hepatol 2012;24:1071-7.

15. Yiran W, Qijin W, Xianzhao W, et al. Global scientific trends on exosome research during 2007-2016: a bibliometric analysis. Oncotarget, 2017.

16. D'Arcangelo D, Giampietri C, Facchiano F, et al. BAMM: a preliminary bibliometric analysis on melanoma manuscripts. Pigment Cell Melanoma Res 2013;26:415-7.

17. Bornmann L, Daniel HD. The state of $h$ index research. Is the $h$ index the ideal way to measure research performance? EMBO Rep 2009;10:2-6.

18. Central Intelligence Agency The world factbook. Secondary central intelligence agency the world factbook. https://www.cia.gov/library/ publications/the-world-factbook/

19. Wang Y, Wang Q, Zhu R, et al. Trends of spinal tuberculosis research (1994-2015): A bibliometric study. Medicine 2016;95:e4923.

20. Secondary.https://en.wikipedia.org/wiki/H-index

21. Bagley SC, White H, Golomb BA. Logistic regression in the medical literature: standards for use and reporting, with particular attention to one medical domain. J Clin Epidemiol 2001;54:979-85.

22. Jia ZJ, Hong B, Chen DM, et al. China's growing contribution to global intracranial aneurysm research (1991-2012): a bibliometric study. PLoS One 2014;9:e91594.

23. Synnestvedt MB, Chen $\mathrm{C}$, Holmes JH. CiteSpace II: visualization and knowledge discovery in bibliographic databases. AMIA Annu Symp Proc 2005:724-8.

24. Casciaro S, Conversano F, Pisani P, et al. New perspectives in echographic diagnosis of osteoporosis on hip and spine. Clin Cases Miner Bone Metab 2015;12:142-50.

25. Lin X, Xiong D, Peng YQ, et al. Epidemiology and management of osteoporosis in the People's Republic of China: current perspectives. Clin Interv Aging 2015;10:1017-33.

26. Yao X, Li D, Pei G. In focus: molecular and cell biology research in China. Nat Rev Mol Cell Biol 2013;14:600-6.

27. Chen Z, Wu B, Zhai X, et al. Basic study for ultrasound-based navigation for pedicle screw insertion using transmission and backscattered methods. PLoS One 2015;10:e0122392.

28. Hans D, Dargent-Molina P, Schott AM, et al. Ultrasonographic heel measurements to predict hip fracture in elderly women: the EPIDOS prospective study. Lancet 1996;348:511-4.

29. Bauer DC, Glüer CC, Cauley JA, et al. Broadband ultrasound attenuation predicts fractures strongly and independently of densitometry in older women. A prospective study. Study of Osteoporotic Fractures Research Group. Arch Intern Med 1997;157:629-34.

30. Hodges PW, Pengel LH, Herbert RD, et al. Measurement of muscle contraction with ultrasound imaging. Muscle Nerve 2003;27:682-92.

31. Hodges PW, Richardson CA. Inefficient muscular stabilization of the lumbar spine associated with low back pain. A motor control evaluation of transversus abdominis. Spine 1996;21:2640-50.

32. Grau T, Leipold RW, Conradi R, et al. Efficacy of ultrasound imaging in obstetric epidural anesthesia. J Clin Anesth 2002;14:169-75.

33. Arzola C, Davies S, Rofaeel A, et al. Ultrasound using the transverse approach to the lumbar spine provides reliable landmarks for labor epidurals. Anesth Analg 2007;104:1188-92.

34. Ungi T, King $F$, Kempston $M$, et al. Spinal curvature measurement by tracked ultrasound snapshots. Ultrasound Med Biol 2014;40:447-54.

35. Wang SF, Teng CC, Lin KH. Measurement of cervical range of motion pattern during cyclic neck movement by an ultrasound-based motion system. Man Ther 2005;10:68-72.

36. Young $\mathrm{H}$, Howey S, Purdie DW. Broadband ultrasound attenuation compared with dual-energy $\mathrm{X}$-ray absorptiometry in screening for postmenopausal low bone density. Osteoporos Int 1993;3:160-4.

37. Manbachi A, Cobbold RS, Ginsberg HJ. Guided pedicle screw insertion: techniques and training. Spine J 2014;14:165-79. 